\title{
Neural Network Approach to Variable Structure Based Adaptive Tracking of SISO Systems
}

\author{
Li-Chen Fu \\ Department of Electrical Engineering \\ Department of Computer Science and Information Engineering \\ National Taiwan University, Taipei, Taiwan, R.O.C. \\ October 16, 1996
}

\begin{abstract}
This paper presents a novel approach to adaptive tracking control of linear SISO systems, which can solve the traditional model reference adaptive control (MRAC) problems. In this approach, a neural network universal approximator is included to furnish an on-line estimate of a function of the state and some signals relevant to the desired trajectory. The salient feature of the present work is that a rigorous proof via Lyapunov stability theory is provided. It is shown that the output error will fall into a residual set which can be made arbitrarily small.
\end{abstract}

\section{Introduction}

In the past two decades, model reference adaptive control (MRAC) has evolved as one of the most soundly developed adaptive control techniques, though several constraints remain to be resolved. Not only the stability property has been rigorously established [11] [10] but also the performance has been made continuously improving [3] [14] [4] [7].

Recently, neural networks have shown great promise in the realm of nonlinear control problems because of their universal approximation capability. This powerful property inspires a great number of neural-network based controllers without significant prior knowledge of the system dynamics. Till now, some developed feedforward neural-network based control schemes, whose network parameters are adapted according to Lyapunov theory, have provided rigorous theoretical analysis. In particular, Sanner and Slotine [13] utilized the spatial sampling theory to design a Gaussian radial basis function network. Based on their work, adaptive control theory can be applied to deal with the plant nonlinearities. Moreover, Chen and Liu [1],
Chen and Khalil [2] used multilayer feedforward networks along with the backpropagation (BP) rule and on-line update rule, respectively, with a deadzone to overcome the modeling errors. Following the similar line of thoughts, Lewis et al. [9] also utilized multilayer feedforward networks, but applied the $e_{1}$-modification to the BP rule and added an extra robustifying control signal.

Despite that there exists quite a few neural-network based controllers for nonlinear systems as mentioned earlier, there are relatively fewer results about applying the similar concept to solve the traditional MRAC problems. By doing so, it in fact can be shown that the need to have some reference model to be followed by the unknown plant, as has been quite often seen in the literature, can be completely relaxed i.e., the class of MRAC problems can simply be reformulated as adaptive tracking problems, and, hence, the so-developed approach apparently will be more general.

In this paper, we propose a novel neural-network approach to solve the traditional MRAC problems. First, a feedforward neural network model [9] (cf. [8]) is established and detailedly analyzed for subsequent controller design. It is shown that a bound on the modeling error can, in fact, be expressed as a sum of a linearly parametrized form and a small residual error. By adopting a neural-network based sliding mode control, it can be shown that the present developed approach does not require the prior knowledge of the so-called "optimal" neural networks, or the "optimal" neural weights, in contrast with all the other approaches mentioned previously. Along with the control law, a stable adaptive law is devised by Lyapunov theory, whereby the boundedness of all signals as well as the convergence of the tracking errors of the closed-loop system are clearly guaranteed. 


\section{Problem Formulation}

Consider an SISO linear system described by

$y_{p}=k_{p} \frac{s^{m}+b_{1} s^{m}-1+\cdots+b_{m}}{s^{n}+a_{1} s^{n}-1+\cdots+a_{n}}\left(u_{p}\right)=k_{p} \frac{\widehat{n}_{p}(s)}{\widehat{d}_{p}(s)}\left(u_{p}\right)$,

where $m \leq n, y_{p}$ amd $u_{p}$ denote the plant output and the plant input, respectively, and $n$ and $m$ are some known positive integers.

Such linear plant does not have to be stable, but the sign of the high frequency gain $k_{p}$ has to be known $a$ priori and the plant has to be minimum-phase, which are stated in the following assumptions:

Assumption (A1). The value of $k_{p}$ may not be known, but its sign should be known. Without loss of generality, throughout the paper we will assume that it is positive here.

Assumption (A2). The plant transfer function described by (1) is minimum-phase, i.e., all its zeros lie in the open left half complex plane.

Assumption (A3). All the coefficients of $\hat{n}_{p}(s)$ and $\widehat{d}_{p}(s)$ are unknown a priori, but they are co-prime.

Let $y_{d}$ denote the desired trajectory to be followed by the plant output $y_{p}$. It is assumed to be at least $(n-m)$ times differentiable and satisfies the following assumption:

Assumption (A4). The signals $y_{d}, y_{d}^{(1)}, \ldots$. , $y_{d}^{(n-m)}$ exist and are uniformly bounded.

Now we are ready to sate the problem to be solved in this paper. Given a linear SISO plant described by eq. (1) satisfying assumptions (A1) - (A3) and given a desired trajectory $y_{d}$ satisfying assumption (A4), devise control input $u_{p}$ using only input and output measurements such that the plant output $y_{p}$ will follow $y_{d}$ as closely as possible.

\section{Feedforward Neural Networks}

In a neural network, the simple nonlinear elements, called neurons, are interconnected, and the strengths of the interconnections are denoted by adjustable parameters called neuron weights. A three-layer feedforward neural network is used to design a robust adaptive neural controller. The structure of the neural network is shown in Fig. 1, which performs as an approximator described in a matrix form as follows:

$$
\widehat{h}_{n}\left(\bar{x}, \widehat{W}_{h}, \widehat{V}_{h}\right)=\widehat{W}_{h} \sigma\left(\widehat{V}_{h} \bar{x}_{a}\right),
$$

where $\widehat{W}_{h} \in \mathcal{R}^{m \times p}$ and $\widehat{V}_{h} \in \mathcal{R}^{p \times(n+1)}$ are outputhidden weight matrix and hidden-input weight matrix, respectively, $\bar{x} \in \mathcal{R}^{n \times 1}$ is the input vector, $\bar{x}_{a} \equiv\left(\bar{x}^{T},-1\right)^{T} \in \mathcal{R}^{(n+1) \times 1}$ is the augmented neural input vector (the -1 term denotes the input bias),

$$
\sigma_{i}\left(\widehat{V}_{h_{i}} \bar{x}_{a}\right) \equiv \frac{1}{1+\exp \left(-\widehat{V}_{h_{i}} \bar{x}_{a}\right)} \in \mathcal{R}, \quad i=1, \cdots, p
$$

is a sigmoid function, and

$\sigma\left(\widehat{V}_{h} \bar{x}_{a}\right) \equiv\left(\begin{array}{c}\sigma_{1}\left(\widehat{V}_{h_{1}} \bar{x}_{a}\right) \\ \vdots \\ \sigma_{p}\left(\hat{V}_{h_{p}} \bar{x}_{a}\right)\end{array}\right)$, with $\widehat{V}_{h} \equiv\left(\begin{array}{c}\widehat{V}_{h_{1}} \\ \vdots \\ \widehat{V}_{h_{p}}\end{array}\right)$,

where $\widehat{V}_{h}$ includes the threshold, $\sigma_{i}\left(\widehat{V}_{h_{i}} \bar{x}_{a}\right) \equiv \widehat{\sigma}_{i}$ for each $i=1, \cdot, p$, and $\sigma\left(\widehat{V}_{h} \bar{x}_{a}\right) \equiv \widehat{\sigma}$.

Theorem 1. Let $\bar{x} \in \mathcal{D}$ (a compact subset of $\mathcal{R}^{n}$ ), $h(\bar{x}): \mathcal{D} \rightarrow \mathcal{R}^{m}$ is a continuous function vector. For an arbitrary constant $\epsilon>0$, there exists an integer $p$ (the number of hidden neurons) and real constant optimal weight matrices $W_{h} \in \mathcal{R}^{m \times p}$ and $V_{h} \in \mathcal{R}^{p \times(n+1)}$ such that

$$
h(\bar{x})=h_{n}\left(\bar{x}, W_{h}, V_{h}\right)+\epsilon_{h}(\bar{x})
$$

where $\epsilon_{h}(\bar{x})$ is the approximation error vector satisfying $\left\|\epsilon_{h}(\bar{x})\right\| \leq \epsilon, \forall \bar{x} \in \mathcal{D}$. The optimal approximator can be described as

$$
h_{n}\left(\bar{x}, W_{h}, V_{h}\right) \equiv W_{h} \sigma\left(V_{h} \bar{x}_{a}\right)
$$

where $\|\cdot\|$ denotes the 2-norm, and $\bar{x}_{a} \equiv\left(\bar{x}^{T},-1\right)^{T}$. Proof: Please see Funahashi [5].

In general, studying the stability of a multilayer neural-network based control system is difficult because the corresponding dynamics are nonlinear in adjustable neural network weights. Such a structure is generally not suitable for adaptive control. Fortunately, the approximation error $\widehat{h}_{n}$ can be expressed in linearly parameterized form modulo a residual term. This is stated in the following lemma.

Lemma 1. [[? Jhang] Define the estimation errors of the weight matrix as:

$$
\widetilde{W}_{h} \equiv W_{h}-\widehat{W}_{h}, \quad \widetilde{V}_{h} \equiv V_{h}-\widehat{V}_{h}
$$

and the estimation error of the hidden layer vector as $\widetilde{\sigma} \equiv \sigma\left(V_{h} \bar{x}_{a}\right)-\sigma\left(\widehat{V}_{h} \bar{x}_{a}\right)$, then the function approximation error $\widetilde{h}_{n}$ is

$$
\begin{aligned}
\widetilde{h}_{n} & \equiv h(\bar{x})-\widehat{h}_{n}\left(\bar{x}, \widehat{W}_{h}, \widehat{V}_{h}\right) \\
& =\widetilde{W}_{h} \cdot\left(\widehat{\sigma}-\widehat{\sigma}^{\prime} \widehat{V}_{h} \bar{x}_{a}\right)+\widehat{W}_{h} \widehat{\sigma}^{\prime} \widetilde{V}_{h} \bar{x}_{a}+d_{h}
\end{aligned}
$$

where the residual term $d_{h}$ can be expressed as

$$
d_{h}=\widetilde{W}_{h} \hat{\sigma}^{\prime} V_{h} \bar{x}_{a}+W_{h} \sigma\left(\tilde{V}_{h} \bar{x}_{a}\right)+\epsilon_{h}(\bar{x}),
$$


with o(.) representing a sum of high order terms of the argument in a Taylor series expansion.

Lemma 2. [[?]hang] The residual term $d_{h}$ can be bounded by a linear-in-parameter function, i.e.,

$$
\left\|d_{h}\right\|<\alpha_{h}^{T} Y_{\alpha}
$$

where the unknown parameter vector $\alpha_{h} \in \mathcal{R}^{4 \times 1}$ is composed of optimal weight matrices and some bounded constants, and the known function vector $Y_{\alpha}$ is equal to $\left(1,\left\|\bar{x}_{a}\right\|,\left\|\bar{x}_{a}\right\|\left\|\widehat{W}_{h}\right\|_{F},\left\|\bar{x}_{a}\right\|\left\|\widehat{V}_{h}\right\|_{F}\right)^{T}$.

\section{Variable Structure Based Adaptive Tracking Controller}

Referring to the SISO plant described by (1), we let $\left(A_{p}, b_{p}, c_{p}\right)$ be its minimal realization given below:

$$
\begin{aligned}
\dot{x}_{p} & =\left(\begin{array}{ccccc}
-a_{n} & 1 & 0 & \cdots & 0 \\
-a_{n-1} & 0 & 1 & \cdots & 0 \\
\vdots & \vdots & \vdots & \cdots & \vdots \\
-a_{1} & 0 & 0 & \cdots & 0
\end{array}\right) x_{p}+\left(\begin{array}{c}
b_{n}^{\prime} \\
b_{n-1}^{\prime} \\
\vdots \\
b_{1}^{\prime}
\end{array}\right) u_{p} \\
& =A_{p} x_{p}+b_{p} u_{p} \\
y_{p} & =\left(\begin{array}{ccccc}
1 & 0 & 0 & \cdots & 0
\end{array}\right) x_{p}=c_{p}^{T} x_{p}
\end{aligned}
$$

where $b_{1}^{\prime}=0, \ldots, b_{n-m-1}^{\prime}=0, b_{n-m}^{\prime}=k_{p}, b_{n-m+1}^{\prime}=$ $k_{p} b_{1}, \ldots, b_{n}^{\prime}=k_{p} b_{m}$. Let $A_{0}$ be a Hurwitz matrix chosen as follows:

$$
A_{0}=\left(\begin{array}{ccccc}
-a_{n}^{\prime} & 1 & 0 & \cdots & 0 \\
-a_{n-1}^{\prime} & 0 & 1 & \cdots & 0 \\
\vdots & \vdots & \vdots & \cdots & \vdots \\
-a_{1}^{\prime} & 0 & 0 & \cdots & 0
\end{array}\right)
$$

which will have the desired property to be clear later on. Then, it is straightforward to derive the following from eq. (5):

$$
\begin{aligned}
\dot{x}_{p} & =A_{0} x_{p}+\left(A_{p}-A_{0}\right) x_{p}+b_{p} u_{p} \\
& =A_{0} x_{p}+\widetilde{a} y_{p}+b_{p} u_{p} \\
y_{p} & =c_{p}^{T} x_{p}
\end{aligned}
$$

where we used the fact that

$$
\begin{aligned}
\left(A_{p}-A_{0}\right) x_{p} & =\left(\begin{array}{lll}
a_{n}^{\prime}-a_{n} & \cdots & a_{1}^{\prime}-a_{1}
\end{array}\right)^{T} c_{p}^{T} x_{p} \\
& =\widetilde{a} c_{p}^{T} x_{p}=\widetilde{a} y_{p}
\end{aligned}
$$

Now, we again adopt the mixed use of a timed signal and a Laplace transfer function to obtain the explicit expression of $x_{p}$ in terms of $y_{p}$ and $u_{p}$ as follows

$$
\begin{array}{r}
x_{p}=\left(s I-A_{0}\right)^{-1} \tilde{a}\left(y_{p}\right)+\left(s I-A_{0}\right)^{-1} b_{p}\left(u_{p}\right)+\epsilon \\
=\frac{1}{\widehat{\lambda}(s)}\left(\begin{array}{c}
\widehat{d}_{11}(s) \\
\vdots \\
\widehat{d}_{1 n}(s)
\end{array}\right)\left(y_{p}\right)+\frac{1}{\widehat{\lambda}(s)}\left(\begin{array}{c}
\widehat{d}_{21}(s) \\
\vdots \\
\widehat{d}_{2 n}(s)
\end{array}\right)\left(u_{p}\right)+\epsilon
\end{array}
$$

where $\hat{\lambda}(s)=\operatorname{det}\left(s I-A_{0}\right)$ is the characteristic polynomial of $A_{0}$ with degree $n, \widehat{d}_{i j}(s)$ are polynomials of $s, i=1,2, j=1,2, \ldots, n$, of which each has degree no greater than $(n-1)$, and $\epsilon$ stands for the exponentially decaying terms to capture the initial condition effect.

On the other hand, define an $n \times n$ Hurwitz matrix $\Lambda$ as:

$$
\Lambda=\left(\begin{array}{ccccc}
-0 & 1 & 0 & \cdots & 0 \\
-0 & 0 & 1 & \cdots & 0 \\
\vdots & & & & \\
-a_{n}^{\prime} & -a_{n-1}^{\prime} & -a_{n-2}^{\prime} & \cdots & -a_{1}^{\prime}
\end{array}\right)
$$

whose characteristic polynomial apparently is the same as that of $A_{0}$. Then, construct two $n$-dim signal vector $\omega_{1}$ and $\omega_{2}$ as follows:

$$
\begin{aligned}
& \dot{\omega}_{1}=\Lambda \omega_{1}+b_{\Lambda} u_{p} \\
& \dot{\omega}_{2}=\Lambda \omega_{2}+b_{\Lambda} y_{p}
\end{aligned}
$$

where $b_{\Lambda}=[0, \ldots, 1]^{T}$, which by some manipulations will lead to explicit expressions of $\omega_{1}$ and $\omega_{2}$, i.e.,

$$
\begin{aligned}
& \omega_{1}=\frac{1}{\widehat{\lambda}(s)}\left(\begin{array}{c}
1 \\
s \\
\vdots \\
s^{n}-1
\end{array}\right)\left(y_{p}\right) \\
& \omega_{2}=\frac{1}{\widehat{\lambda}(s)}\left(\begin{array}{c}
1 \\
s \\
\vdots \\
s^{n}-1
\end{array}\right)\left(u_{p}\right)
\end{aligned}
$$

By comparing (6) and (7),(8), we can easily conclude that

$$
x_{p}=T_{1} \omega_{1}+T_{2} \omega_{2}+\epsilon
$$

for some proper $n \times n$ constant matrices $T_{1}$ and $T_{2}$.

Let $e_{0}$ denote the output tracking error, i.e., $e_{0}=$ $y_{p}-y_{d}$, and define a sliding surface variable $S$ as :

$$
S=e_{0}^{(r-1)}+\lambda_{1} e_{0}^{(r-2)}+\cdots+\lambda_{r-2} e_{0}^{(1)}+\lambda_{r-1} e_{0}
$$

where $r=n-m$ and $\lambda_{i}, i=1,2, \ldots, r-1$, are chosen such that the polynomial

$$
\begin{aligned}
\widehat{P}(s) & =s^{r-1}+\lambda_{1} s^{r-2}+\cdots+\lambda_{r-2} s+\lambda_{r-1} \\
& =\prod_{i=1}^{r-1}\left(s+\alpha_{i}\right)=\prod_{i=1}^{r-1} \widehat{l}_{i}(s)
\end{aligned}
$$


is Hurwitz, for some $\alpha_{i}>0, i=1,2, \ldots, r-1$. Obviously, $S=\hat{P}(s)\left(e_{o}\right)$. Using the fact that

$$
\begin{aligned}
& y_{p}^{(1)}=c_{p}^{T} A_{p} x_{p}, \cdots y_{p}^{(r-1)}=c_{p}^{T} A_{p}^{r-1} x_{p} \\
& y_{p}^{(r)}=c_{p}^{T} A_{p}^{r} x_{p}+c_{p}^{T} A_{p}^{r-1} b_{p} u_{p}=c_{p}^{T} A_{p}^{r} x_{p}+k_{p} u_{p}
\end{aligned}
$$

we can obtain the following equality:

$$
\dot{e}_{o}=-\frac{1}{2} \bar{M} e_{o \Delta}+k_{p}\left(\phi(\bar{x})+\frac{1}{\hat{P}(s)}\left(u_{p}\right)\right)
$$

for some $\bar{M}>0$, where $\bar{x}=\left[\omega_{f 1}^{T}, \omega_{f 2}^{T}, Y_{f d}\right]^{T}$ with $\omega_{f 1}=\frac{1}{\hat{P}(s)}\left(\omega_{1}\right), \omega_{f 2}=\frac{1}{\hat{P}(s)}\left(\omega_{2}\right)$, and $Y_{f d}=\frac{1}{\hat{P}(s)}\left(Y_{d}\right)$, and $e_{o \Delta}$ will be defined later in the sequel.

To proceed with the controller design, we define a set of auxiliary error signals:

$$
\begin{aligned}
e_{1} & =\frac{1}{l_{1}(s)}\left(u_{p}\right)-\frac{1}{F(\tau s)}\left(u_{1}\right) \\
e_{2} & =\frac{1}{l_{2}(s)}\left(u_{1}\right)-\frac{1}{F(\tau s)}\left(u_{2}\right) \\
& \vdots \\
e_{r-1} & =\frac{1}{l_{r-1}(s)}\left(u_{2}\right)-\frac{1}{F(\tau s)}\left(u_{r-1}\right)
\end{aligned}
$$

where $F(\tau s)$ is any Hurwitz polynomial in $\tau s$ for some $\tau \geq 0$, with degree at least two and $F(0)=1$. In the literature, $1 / F(\tau s)$ is referred to as an averaging filter, which is obviously a low-pass filter whose bandwidth can be arbitrarily enlarged as $\tau \rightarrow 0$. In other words, if $\tau$ is smaller and smaller, the filter $1 / F(\tau s)$ is flatter and flatter.

Rewrite eq. (11) as:

$$
\begin{aligned}
\dot{e}_{1} & =-\alpha_{1} e_{1}+u_{p}-\frac{\hat{l}_{1}(s)}{\hat{F}(\tau s)}\left(u_{1}\right) \\
\dot{e}_{2} & =-\alpha_{1} e_{2}+u_{1}-\frac{\hat{l}_{2}(s)}{\hat{F}(\tau s)}\left(u_{2}\right) \\
& \vdots \\
\dot{e}_{r-1} & =-\alpha_{r-1} e_{r-1}+u_{r-2}-\frac{\hat{l}_{r-1}(s)}{\hat{F}(\tau s)}\left(u_{r-1}\right)(11)
\end{aligned}
$$

and note that

$\left|\frac{\hat{l}_{j}(s)}{\hat{F}(\tau s)}\left(u_{j}(t)\right)\right| \leq \frac{1}{2}\left\|\left(u_{j}\right)_{t}\right\|_{\infty}, j=1,2, \ldots, r-1, \forall t \geq 0$

where $\left\|(\cdot)_{t}\right\|_{\infty}$ denotes the truncated sup-norm. The signals $u_{i}, i=1,2, \ldots, r-1$, are called auxiliary compensation signas to be defined later.
By some observation of the above set of error equations (11), if we define the signals $u_{i}, i=0,1, \ldots, r-2$, where $u_{0}=u_{p}$, as follows:

$$
\begin{aligned}
u_{0} & =-\operatorname{sgn}\left(e_{1}\right)\left(\frac{1}{2}\left\|\left(u_{1}\right)_{t}\right\|_{\infty}+\eta\right) \\
u_{1} & =-\operatorname{sgn}\left(e_{2}\right)\left(\frac{1}{2}\left\|\left(u_{2}\right)_{t}\right\|_{\infty}+\eta\right) \\
& \vdots \\
u_{i-1} & =-\operatorname{sgn}\left(e_{i}\right)\left(\frac{1}{2}\left\|\left(u_{i}\right)_{t}\right\|_{\infty}+\eta\right) \\
& \vdots \\
u_{r-2} & =-\operatorname{sgn}\left(e_{r-1}\right)\left(\frac{1}{2}\left\|\left(u_{r-1}\right)_{t}\right\|_{\infty}+\eta\right)
\end{aligned}
$$

for some $\eta>0$, then we can readily conclude that $e_{i}$, $i=1,2, \ldots, r-1$, will reach the zero value in finite time using the following argument:

$$
\frac{d}{d t}\left|e_{i}\right| \leq-\alpha_{i}\left|e_{i}\right|-\eta
$$

when $\left|e_{i}\right| \neq 0, i=1,2, \ldots, r-1$. Thus, we can view (12) equivalently as

$$
u_{p}=\frac{\widehat{l}_{1}(s) \cdots \widehat{l}_{r-1}(s)}{\hat{F}(s)^{r-1}}\left(u_{r-1}\right)=\frac{\hat{P}(s)}{\hat{F}(s)^{r-1}}\left(u_{r-1}\right)
$$

after some fininte period of time. Referring back to (10), we can rewrite it as

$$
\begin{aligned}
\dot{e}_{o} & =-\frac{1}{2} \bar{M} e_{o \Delta}+k_{p}\left(\phi(\bar{x})+\frac{1}{\hat{F}(s)^{r-1}}\left(u_{r-1}\right)\right) \\
& =-\frac{1}{2} \bar{M} e_{o \Delta}+k_{p}\left(\phi(\bar{x})+u_{r-1}+\delta_{\tau}(t)\right)
\end{aligned}
$$

where $\delta_{\tau}=\hat{G}(\tau s)\left(u_{r-1}\right)=-\left(1-\frac{1}{\hat{F}(\tau s)^{r-1}}\right)\left(u_{r-1}\right)$ satisfies

$$
\left|\delta_{\tau}(t)\right| \leq\left|\hat{G}(\tau s)\left(u_{r-1}(t)\right)\right| \leq r f_{c}\left\|\left(u_{r-1}\right)_{t}\right\|_{\infty}
$$

for some $f_{c} \geq 0$.

By Theorem 1 and Lemma 1, we assume that there exists an optimal neural approximator $\phi_{n}=$ $W_{\phi} \sigma\left(V_{\phi} \bar{x}_{a}\right)$ of the function $\phi(\bar{x})$ and then design a neural approximator $\widehat{\phi}_{n}\left(\widehat{W}_{\phi}, \widehat{V}_{\phi}, \bar{x}_{a}\right)$ to model such function $\phi(\bar{x})$, and the approximation error $\widetilde{\phi}_{n}$ is

$$
\begin{aligned}
\tilde{\phi}_{n}\left(\widehat{W}_{\phi}, \widehat{V}_{\phi}, \bar{x}_{a}\right) & \equiv \phi-\widehat{\phi}_{n} \\
& =\widetilde{W}_{\phi} \cdot\left(\hat{\sigma}-\widehat{\sigma}^{\prime} \widehat{V}_{\phi} \bar{x}_{a}\right)+\widehat{W}_{\phi} \hat{\sigma}^{\prime} \widetilde{V}_{\phi} \bar{x}_{a}+d_{\phi}
\end{aligned}
$$


where $\bar{x}_{a} \equiv\left(\bar{x}^{T},-1\right)^{T} \in \mathcal{R}^{(2 n+2)}$ is the augmented neural input vector, $\widetilde{W}_{\phi} \equiv W_{\phi}-\widehat{W}_{\phi}, \widetilde{V}_{\phi} \equiv V_{\phi}-\widehat{V}_{\phi}$ are the estimation errors of $W_{\phi}$ and $V_{\phi}$, respectively. For clarity, we rewrite all the variables explicitly as follows:

$$
\begin{aligned}
\widehat{V}_{\phi} & =\left(\widehat{V}_{\phi_{1}}^{T}, \cdots, \widehat{V}_{\phi_{p}}^{T}\right)^{T} \in \mathcal{R}^{p \times(2 n+2)} \\
\widehat{W}_{\phi} & =\left(\widehat{W}_{\phi_{1}}, \cdots, \widehat{W}_{\phi_{p}}\right) \in \mathcal{R}^{1 \times p} \\
\widehat{\sigma} & =\left(\sigma_{1}\left(\widehat{V}_{\phi_{1}} \bar{x}_{a}\right), \cdots, \sigma_{p}\left(\widehat{V}_{\phi_{p}} \bar{x}_{a}\right)\right) \in \mathcal{R}^{p \times 1} \\
\widehat{\sigma}^{\prime} & =\operatorname{diag}\left\{\sigma_{1}^{\prime}\left(\widehat{V}_{\phi_{1}} \bar{x}_{a}\right), \cdots, \sigma_{p}^{\prime}\left(\widehat{V}_{\phi_{p}} \bar{x}_{a}\right)\right\} \in \mathcal{R}^{p \times p}
\end{aligned}
$$

Furthermore, from Lemma 2 the upper bound of the residual term $d_{\phi}$ can be found as follows:

$$
\left\|d_{\phi}\right\| \leq \alpha_{\phi}^{T} Y_{\alpha}
$$

where $\alpha_{\phi} \in \mathcal{R}^{4 \times 1}$ is an unknown parameter vector, and $Y_{\alpha} \in \mathcal{R}^{4 \times 1}$ is a known vector defined to be

$$
Y_{\alpha}=\left(1,\left\|\bar{x}_{a}\right\|,\left\|\bar{x}_{a}\right\|\left\|\widehat{W}_{\phi}\right\|_{F},\left\|\bar{x}_{a}\right\|\left\|\widehat{V}_{\phi}\right\|_{F}\right)^{T}
$$

Consequently, the control law $u_{r-1}$ is designed as

$$
\begin{aligned}
u_{r-1} & =-\widehat{\phi}_{n}+v_{p} \\
v_{p} & =-\operatorname{sat}\left(e_{o} / \varepsilon\right)\left(\widehat{\alpha}_{\phi}^{T} Y_{\alpha}+\beta_{0}\right),
\end{aligned}
$$

for some $\beta_{0} \geq 0$, where a deadzone size $\varepsilon$ is used to avoid discontinuous control transitions, and sat $(\cdot)$ is the saturation function and the adaptive law is described as

$$
\begin{aligned}
\dot{\widehat{W}}_{\phi}^{T} & =\Gamma_{w} \cdot\left(\widehat{\sigma}-\widehat{\sigma}^{\prime} \widehat{V}_{\phi} \bar{x}_{a}\right) e_{o \Delta} \\
\dot{\hat{V}}_{\phi}^{T} & =\Gamma_{v} \cdot\left(\bar{x}_{a} e_{o \Delta} \widehat{W}_{\phi} \widehat{\sigma}^{\prime}\right) \\
\dot{\alpha}_{\phi} & =\Gamma_{\alpha}\left|e_{o \Delta}\right| Y_{\alpha},
\end{aligned}
$$

where the adaptive gain matrices $\Gamma_{w}, \Gamma_{v}$, and $\Gamma_{\alpha}$ are all symmetric positive definite with

$$
e_{0 \Delta}=e_{0}-\varepsilon \operatorname{sat}\left(e_{0} / \varepsilon\right)
$$

being used to carry out a smooth adaptive law with a deadzone size $\varepsilon$. Such a deadzone technique has often been incorporated to deal with the phenomenon of parameter drift in robust adaptive control theory [11]. Note that the function $e_{0 \Delta}$ has several useful properties as listed below:

- If $\left|e_{0}\right| \leq \varepsilon$, then $e_{0 \Delta}=0=\dot{e}_{0 \Delta}$

- If $\left|e_{0}\right|>\varepsilon$, then $\left|e_{0 \Delta}\right|=\left|e_{0}\right|-\varepsilon$ and $\dot{e}_{o \Delta}=\dot{e}_{o}$

- $\quad e_{0 \Delta} \operatorname{sat}\left(e_{0} / \varepsilon\right)=\left|e_{0 \Delta}\right|$
The block diagram of the proposed controller is shown in Fig. 2, and the effect of this controller is stated via the following theorem.

Theorem 2. Consider the SISO system (1) satisfying Assumptions $(A 1)-(A 4)$. Define the control law as given in (14) and the adaptive law as in (15). If $y_{d}, \cdots$, $y_{d}^{(r)}$ are all bounded, then for sufficient number of hidden neurons the tracking error $e \equiv y-y_{d}$ will asymptotically converge to a neighborhood of zero whose size is of an order of $\varepsilon$, and all adjustable parameters as well as the internal signals will remain bounded.

Before we prove Theorem 2, we first present the following useful lemma.

Lemma 3. Let $u_{p}$ be the variable defined in eq. (12), then it follows that

$$
\left\|\left(u_{p}\right)_{t}\right\|_{\infty} \leq \beta_{1}\left\|\left(e_{o}\right)_{t}\right\|_{\infty}+\beta_{2}, \forall t \geq 0
$$

for some $\beta_{1}, \beta_{2} \geq 0$, provided that $\widehat{V}_{\phi}, \widehat{W}_{\phi}$, and $\alpha_{\phi}$ are uniformly bounded.

Proof of Theorem 2: From the definition and property of the function, $\phi(\cdot)$, in equation (9), Theorem 1 guarantees the existence of a sufficiently large compact set $\mathcal{D}$ (enclosing the origin) and an optimal neural network with approximation accuracy $\epsilon$ over the set $\mathcal{D}$ and satisfying the Remark 1. Now, suppose the initial involved state is well within the compact set $\mathcal{D}$, and then choose a Lyapunov function $V=V_{1}+V_{2}$, where

$$
\begin{aligned}
V_{1} & =\frac{1}{2} e_{o \Delta}^{2} \\
V_{2} & =\frac{1}{2} \widetilde{W}_{\phi} \Gamma_{w}^{-1} \widetilde{W}_{\phi}^{T}+\frac{1}{2} \operatorname{tr}\left\{\widetilde{V}_{\phi} \Gamma_{v}^{-1} \widetilde{V}_{\phi}^{T}\right\}+\frac{1}{2} \widetilde{\alpha}_{\phi}^{T} \Gamma_{\alpha}^{-1} \widetilde{\alpha}_{\phi}
\end{aligned}
$$

According to the properties of $e_{o \Delta}$ and the adaptive law (14), it follows that $V=V_{2}$ and $\dot{V}=0$ for $\left|e_{o}\right| \leq \varepsilon$. In the case where $\left|e_{o}\right|>\varepsilon$, the time derivative of $V_{1}$ can be found referring to the result of Lemma 2 as

$$
\begin{aligned}
\dot{V}_{1}= & e_{o \Delta} \dot{e}_{o} \\
\leq & \widetilde{W}_{\phi} \cdot\left(\widehat{\sigma}-\widehat{\sigma}^{\prime} \widehat{V}_{\phi} \bar{x}_{a}\right) e_{o \Delta}+\operatorname{tr}\left\{\tilde{V}_{\phi} \bar{x}_{a} e_{o \Delta} \widehat{W}_{\phi} \widehat{\sigma}^{\prime}\right\} \\
& +\left|e_{o \Delta}\right| \widetilde{\alpha}_{\phi}^{T} Y_{\alpha}-\left|e_{o \Delta}\right|\left(\beta_{0}-\delta_{\tau}(t)\right)-\frac{1}{2} \bar{M} e_{o \Delta}^{2}
\end{aligned}
$$

Now, evaluating the time derivative of $V_{2}$, we obtain

$$
\dot{V}_{2}=-\widetilde{W}_{\phi} \Gamma_{w}^{-1} \dot{\widehat{W}}_{\phi}^{T}-\operatorname{tr}\left\{\widetilde{V}_{\phi} \Gamma_{v}^{-1} \dot{\hat{V}}_{\phi}^{T}\right\}-\widetilde{\alpha}_{\phi}^{T} \Gamma_{\alpha}^{-1} \dot{\hat{\alpha}}_{\phi}
$$

which, along with the adaptive law (14), leads to the following: 
provided that $\delta_{\tau}(t) \leq \beta_{0}$ for $t \in[0, T]$ for some positive $T$. From the above and Lemma 3, we know that $e_{o}$ and, hence, $u_{p}$ are uniformly bounded over $[0, T]$, which in turn implies that the whole state $X$ defined in Lemma 3 is also uniformly bounded. As a result, $\delta_{\tau}$ is uniformly bounded as well. By letting $\tau$ to be chosen small enough so that $\left|\delta_{\tau}\right|$ can be arbitrarily made smaller, it can then be verified that eq. (16) will hold for all time $t \geq 0$, and therefore

$$
-\int_{0}^{\infty} \dot{V} d t<\infty \text { implies } e_{o \Delta} \in L_{2}
$$

Note that all the derivations so far are independent of the set $\mathcal{D}$ and, hence, the foregoing analysis and boundedness conclusion can be easily justified by taking the set $\mathcal{D}$ large enough. Finally, because of the boundedness of $x_{p}$ and $x_{a}$, it is easy to obtain the fact that $Y_{\alpha}, v_{p}, u_{p}$, and $\dot{e}_{o \Delta}$ are all bounded. As a result of Barbalat's Lemma, we can conclude that $e_{o \Delta} \rightarrow 0$ as $t \rightarrow \infty$, which thus implies the ultimate boundedness of the tracking errors.

Q.E.D.

\section{Conclusions}

In this paper, a novel approach using neural networks to solve the adaptive tracking problem for linear SISO systems was proposed. First, a feedforward neural network with sigmoid hidden units were detailedly analyzed for subsequent controller design. Based on the above-mentioned analytic results, an online tuning multilayer neural-network based controller has been developed. This scheme combines neural networks and sliding mode control technique, where the former is mainly applied to model some unknown functions of inaccessible states whereas the latter is used to overcome some modeling residual term. It has been shown that this approach can solve traditional MRAC problems but do not require the a priori setting of the reference model. Rigorous proof using Lyapunov stability theory was provided. Another salient feature is the the controller design never requires the unrealistic information on the optimal neural network (e.g. the upper bound on the norm of the optimal weight matrices)

\section{References}

[1] Chen, F.-C. and C.-C. Liu, "Adaptively Controlling Nonlinear Continuous-Time Systems Using Multilayer Neural Networks," IEEE Trans. Automatic Control, vol. 39, no. 6, pp. 1306-1310, 1994.
[2] Chen, F.-C. and H.K. Khalil, "Adaptive Control of a Class of Nonlinear Discrete-Time Systems Using Neural Networks," IEEE Trans. on Automatic Control, vol. 40, no 5, pp. 791-801, 1995.

[3] Datta, A. and Ioannou, P.A., "Performance Improvement versus Robust Stability in Model Reference Adaptive Control," Proc. CDC, pp. 748$753,1991$.

[4] Fu, L.-C., "A Robust Model Reference Adaptive Control Using Variable Structure Adaptation for a Class of Plants," Int. J. Control, vol. 53, pp. $1359-1375,1991$.

[5] Funahashi, K.-I., "On the Approximate Realization of Continuous Mappings by Neural Networks," Neural Networks, vol. 2, pp. 183-192, 1989.

[6] Hornik, K., M. Stinchcombe and H. White, "Multilayer Feedforward Networks are Universal Approximators," Neural Networks, vol. 2, pp. 359$366,1989$.

[7] Hsu, L. and R.R. Costa, "Variable Structure Model Reference Adaptive Control Using Only Input and Output Measurement: Part 1," Int. J. Control, vol. 49, pp. 339-419, 1989.

[8] Kosmatopoulos, E.B., M.M Polycarpou, M.A. Christodoulou, and P.A. Ioannou, "High-Order Neural Network Structures for Identification of Dynamical Systems," IEEE Trans, on Neural Networks, vol. 6, no. 2, pp. 422-431, 1995.

[9] Lewis, F. L., A. Yesildirek and K. Liu, "Neural Net Robot Controller : Structure and Stability proofs," IEEE Conf. Decision and Contr., pp. 2785-2791, 1993

[10] Narendra, K.S. and L. Valavani, "Stable Adaptive Controller Design - Direct Control," IEEE Trans. Automatic Control, Vol. AC-23, pp. 570$583,1978$.

[11] Annaswamy, A. M. and K. S. Narendra Stable Adaptive Systems, Prentice-Hall, 1989.

[12] Narendra, K. S. and S. Mukhopadhyay, "Adaptive Control of Nonlinear Multivariable Systems Using Neural Networks," Neural Networks, vol. 7 , no. 5, pp. 737-752, 1994

[13] Sanner, R. M. and J. J. E. Slotine, "Gaussian Network for Direct Adaptive Control," IEEE Trans. Neural Network, vol. 3, no. 6, pp. 837-863, 1992.

[14] Sun, J., "A Modified Model Reference Adaptive Control Scheme for Improved Transient Performance," Proc. American Control Conference, pp. $150-155,1991$.

[15] Utkin, V.I., Sliding Modes in Control and Optimization, Springer-Verlag, 1992. 